\title{
JNK is required for maintaining the tumor-initiating cell-like properties of acquired chemoresistant human cancer cells
}

\author{
Yuan LIU ${ }^{1, \#}$, Xia ZHANG ${ }^{1,2, \#, ~ J u a n ~ W A N G ~}{ }^{1}$, Jun YANG ${ }^{1}$, Wen-fu TAN ${ }^{1, *}$ \\ ${ }^{1}$ Department of Pharmacology, School of Pharmacy, Fudan University, Shanghai 201203, China; ${ }^{2}$ Department of Pediatric \\ Endocrinology and Genetic Metabolism, Xinhua Hospital, Shanghai Institute for Pediatric Research, Shanghai Jiao Tong University \\ School of Medicine, Shanghai 200092, China
}

\begin{abstract}
Aim: Many studies reveal an association between the acquired chemoresistant phenotype of cancer cells and tumor-initiating celllike properties. The aim of this study was to determine the impact of c-Jun N-terminal kinase (JNK) on the tumor-initiating cell-like properties of acquired chemoresistant human cancer cells.

Methods: Two well-established human acquired chemoresistant cancer cell lines K562/A02 and KB/VCR, as well as their respective parental counterparts K562 and KB were tested. The expression of relevant mRNAs and proteins was detected using qRT-PCR and Western blotting, respectively. Sphere formation and self-renewal assays were used to study the tumor-initiating cell-like properties. Soft agar and colony formation assays were used to investigate tumorigenic ability.

Results: We observed that suppressing JNK activity by its specific small molecule inhibitor SP600125 or by limiting JNK1/2 expression with JNK1/2 shRNA lentiviruses inhibited the expression of pluripotent stem cell markers such as Oct4, Sox2, and Nanog in KB/VCR cells and K562/A02 cells as well as sphere formation and self-renewal abilities of K562/A02 cells. Additionally, inhibition of JNK activity significantly inhibited the in vitro and in vivo tumor-initiating abilities of KB/VCR cells. Furthermore, our data suggest that blocking JNK activity abundantly inhibited the Hedgehog (Hh) pathway activity, as reflected by reduction of Hedgehog (Hh) pathway target genes Gli1 and ptch1 at the mRNA level as well as Gli-luciferase activity.

Conclusion: JNK maintains the tumor-initiating cell-like properties of acquired chemoresistant K562/A02 and KB/VCR cells potentially through activating the Hedgehog pathway. Thus, disruption of tumor-initiating cell-like properties by targeting JNK may be a new approach to combating acquired chemoresistance.
\end{abstract}

Keywords: drug therapy; JNK mitogen-activated protein kinases; K562 cells; KB cells; Hedgehog; tumor-initiating cell-like properties

Acta Pharmacologica Sinica (2015) 36: 1099-1106; doi: 10.1038/aps.2015.58; published online 3 Aug 2015

\section{Introduction}

Despite the great advances that have been made in molecular targeted therapy, chemotherapy using traditional cytotoxic drugs is still a useful avenue for cancer therapies. A major obstacle for successful chemotherapy is acquired drug resistance, namely, multiple-drug resistance ${ }^{[1]}$. To combat acquired chemoresistance and improve chemotherapy efficiency, substantial efforts have been devoted to elucidate the mechanisms underlying chemoresistance. A variety of molecular events are associated with acquired chemoresistance including drug efflux pumps, detoxifying enzyme activation, and apoptotic

\footnotetext{
\# These authors contributed equally to this study.

* To whom correspondence should be addressed.

E-mail wftan@fudan.edu.cn

Received 2015-03-16 Accepted 2015-06-03
}

defects $^{[2]}$. Cytotoxic anti-cancer drugs, which may efficiently debulk tumor mass, often fail to eradicate the tumor and consequently promote cancer relapse. This phenomenon is most likely due to a minor subpopulation of tumor-initiating cells within the tumor ${ }^{[3]}$. Tumor-initiating cells, also designated as cancer stem cells, are characterized by their indefinite selfrenewal ability while maintaining their ability to generate both tumorigenic and non-tumorigenic cells. These cells possess a chemoresistance phenotype, likely due to their enhanced ability for DNA repair, decreased apoptosis, and elevated expression of ATP-binding cassette transporters ${ }^{[3]}$. On the other hand, our lab and other laboratories have demonstrated that acquired chemoresistant cancer cells possess multiple tumorinitiating cell-like characteristics ${ }^{[4-6]}$. We further report that the Hedgehog $(\mathrm{Hh})$ pathway activity is critical for maintaining the tumor-initiating cell-like properties of acquired chemo- 
resistance ${ }^{[6]}$. These findings suggest that the tumor-initiating cell-like properties that are possessed by the acquired chemoresistant cancer cells play a critical role in maintaining the chemoresistance phenotype that occurs during chemotherapy, thereby raising the possibility of combatting acquired chemoresistance via disrupting the tumor-initiating properties of acquired chemoresistant cancer cells. In this regard, it is important to identify feasible molecular targets for abolishing the tumor-initiating cell-like properties of acquired chemoresistant cancer cells, as it may be useful for combating acquired chemoresistance and subsequently improve chemotherapy efficiency.

The c-Jun N-terminal kinases (JNKs), which are key members of the mitogen-activated protein kinase (MAPK) family, are encoded by the three genes JNK1, JNK2, and JNK3. Among them, JNK1 and JNK2 are widely expressed, while JNK3 is specifically expressed in neurons ${ }^{[7]}$. JNKs can be activated by environmental stresses, mitogens, and oncogenes, and they play a crucial role in various biological events such as proliferation, differentiation, survival and migration via activating the downstream effector activating protein 1 (AP1) including c-Jun, JunB, and JunD ${ }^{[8]}$. JNK signaling has been implicated in numerous aspects of cancer progression such as the initiation, proliferation, survival and metastasis of cancers as well as the occurrence of acquired chemoresistance during chemotherapy ${ }^{[8,9]}$. However, whether and how JNK signaling may maintain the tumor-initiating cell-like properties of acquired chemoresistant cancer cells remains to be fully elucidated. We have previously demonstrated that JNK is involved in signal transduction of the Hh pathway ${ }^{[10]}$. In this study, we determined that suppression of JNK activity may abolish the tumor-initiating cell-like properties of acquired chemoresistant cancer cells potentially through inhibiting $\mathrm{Hh}$ pathway activity, thus paving the way for reversal of acquired chemoresistance by targeting JNK.

\section{Materials and methods}

\section{Drugs and antibodies}

Doxorubicin (Dox), Vincristine (VCR), and SP600125 were purchased from Sigma-Aldrich (St Louis, MO, USA). Rabbit monoclonal antibodies for Oct4, Sox2, and Nanog were purchased from Bioss Corporation (Woburn, MA, USA). Rabbit monoclonal antibodies against phospho-c-Jun, JNK1/2, GFP, Flag, and GAPDH were purchased from Cell Signaling Technology (Beverly, MA, USA).

\section{Cell culture}

The K562 human chronic myelogenous leukemia cell line and KB human epidermoid carcinoma cell lines were purchased from the American Type Culture Collection (Manassas, VA, USA) and were cultured according to the manufacturer's instructions. The Dox-selected multidrug-tolerant K562/A02 subline and the VCR-selected multidrug-tolerant KB/VCR subline were cultured as previously described ${ }^{[6]}$. These two resistant cell lines were authenticated by comparing their fold resistances with those of the parental cells and examining $A B C$ transporter (MDR1 and BCRP) expression. The K562/A02 and KB/VCR cells were grown in the absence of Dox or VCR for at least 5-7 d prior to experiments to avoid drug-associated secondary effects ${ }^{[5]}$.

\section{Plasmid and lentivirus transfection}

The pCDNA3 Flag JNK1(APF) plasmids was purchased from Addgene (Cambridge, MA, USA) and engineered into pLVXEGFP-3FLAG-Puro lentivectors. JNK1/2 shRNA harbored by pLVX-EGFP-3FLAG-Puro lentivectors were purchased from Sunbio (Shanghai, China).

Transient transfections were performed using Lipofectamine 2000 reagent (Invitrogen; Grand Island, NY, USA) according to the manufacturer's instructions. The lentiviruses were prepared according to previously reported methods ${ }^{[10]}$.

\section{Quantitative reverse transcription PCR (qRT-PCR)}

After various treatments as indicated, cells were collected for extracting total RNA by RNAiso Plus Kit from TaKaRa (Dalian, China) according to the instructions provided by the manufacturer. The samples were then processed directly to cDNA by reverse transcription using a SuperScript III kit (TaKaRa). Quantitative PCR assays were performed in triplicate with a SYBR-Green kit (TaKaRa) in iCycler iQ system (Bio-Rad; Hercules, CA, USA). The mRNA levels of interested genes were normalized to that of TATA.

Primers for the genes tested were obtained from Invitrogen (Grand Island, NY, USA):

Oct4: 5'-ATTCAGCCAAACGACCATCT-3', 5'-TTGCCTCTCCACTCGGTTCTC-3';

Sox2: 5'-ACACCAATCCCATCCACACT-3', 5'-GCAAA CTTCCTGCAAAGCTC-3';

Nanog: 5'-GTCCCGGTCAAGAAACAGAA-3', 5'-TGCGTCACACCATTGCTATT-3';

Gli1: 5'-GTGGGAAAGGTCTGGGATGT-3', 5'-TGCGCCTGTCTCAGAGTAAAA-3';

Ptch1: 5'-CCACAGAAGCGCTCCTACA-3', 5'-CTGTAATTTCGCCCCTTCC-3';

TATA: 5'-ACCCTTCACCAATGACTCCTATG-3', 5'-TGACTGCAGCAAATCGCTTGG-3'.

\section{Sphere-formation assay and self-renewal assay}

Cells were seeded into ultra-low adherent 96-well plates (Corning; Lowell, MA, USA) at 1000 cells/well in DMEM/F12 medium (Invitrogen; Grand Island, NY, USA) containing B-27 (Invitrogen; Grand Island, NY, USA), $2 \mathrm{mmol} / \mathrm{L}$ glutamine, $10 \mathrm{ng} / \mathrm{mL}$ human epidermal growth factor (Sigma-Aldrich, St Louis, MO, USA), $10 \mathrm{ng} / \mathrm{mL}$ basic fibroblast growth factor (Sigma-Aldrich), $0.5 \mathrm{mg} / \mathrm{mL}$ hydrocortisone and $10 \mathrm{mg} / \mathrm{mL}$ insulin. The culture medium was refreshed every three days. After routine culture for $15 \mathrm{~d}$, spheres were counted under a microscope $\mathrm{e}^{[11]}$. The spheres were collected, dissociated into single cells, and re-plated into ultra-low attachment 96-well plates for determining their self-renewal ability. The number of spheres was counted as described above after culture under the same conditions for $15 \mathrm{~d}$. 


\section{Clonogenic assay}

KB and KB/VCR cells were seeded at approximately 1000 cells in $10-\mathrm{cm}$ dishes. The culture medium that had been supplemented with SP600125 or not was refreshed every three days. After culture for $10 \mathrm{~d}$, the cells were fixed with $4 \%$ paraformaldehyde in PBS, stained with crystal violet solution and photographed.

\section{Soft agar assay}

Cells were harvested and suspended in culture medium. One milliliter of $0.5 \%$ agar (Invitrogen; Grand Island, NY, USA) was added to 6-well plates to make the bottom layer. Next, $500 \mu \mathrm{L} 0.5 \%$ agar was mixed with $500 \mu \mathrm{L}$ cell suspension containing 5000 cells. This mixture was added above the bottom layer and allowed to solidify at room temperature, followed by adding $2 \mathrm{~mL}$ culture medium to the top layer. After routine incubation for 2 weeks, the colonies were photographed.

\section{Western blot analysis}

Cells after transfections were lysed in lysis buffer $(50 \mathrm{mmol} / \mathrm{L}$ Tris, pH 7.4, $150 \mathrm{mmol} / \mathrm{L} \mathrm{NaCl}, 1 \% \mathrm{NP}-40,1 \mathrm{mmol} / \mathrm{L}$ sodium vanadate, $1 \mathrm{mmol} / \mathrm{L}$ PMSF, $1 \mathrm{mmol} / \mathrm{L}$ DTT and $10 \mathrm{mg} / \mathrm{mL}$ leupeptin and aprotinin) and subjected to immunoblot analysis. Various primary antibodies as indicated were used for immunoblot analysis according to the standard procedure.

\section{Dual-luciferase reporter assay}

Cells were plated into 48 -well plates and cotransfected with $8 \times$ Gli binding site luciferase expression constructs and Renilla luciferase plasmids using Lipofectamine 2000 reagent (Invitrogen; Grand Island, NY, USA). Luciferase activities present in cellular lysates were measured using a dual-luciferase reporter assay system from Promega (Madison, WI, USA) and a luminometer (Molecular Device; Sunnyvale, CA, USA). Firefly luciferase values were normalized to Renilla values.

\section{Tumorigenesis assay}

Aliquots of $2 \times 10^{3} \mathrm{~KB}$ or KB/VCR cells that had been infected with GFP, JNK DM, or JNK1/2 shRNA lentiviruses were injected subcutaneously into nude mice (Vital River; Beijing, China). Tumor incidence was monitored for $45 \mathrm{~d}$ after injection. All of the procedures were pre-approved by the Animal Care and Use Committee of Fudan University and performed according to institutional policies.

\section{Statistical analysis}

The data are expressed as the mean $\pm S D$. Statistical analysis was performed by Student's $t$-test. $P<0.05$ was considered statistically significant.

\section{Results}

Inhibition of JNK activity suppresses the expression of stem cells markers in acquired chemoresistant cancer cells

To determine whether JNK interference may disrupt the tumor-initiating cell-like properties of acquired chemoresistant cancer cells, we utilized SP600125, a specific small molecule
JNK inhibitor ${ }^{[12]}$ to inhibit JNK activity. We first examined the effect of SP600125 on the expression of pluripotent stem cell markers such as Oct4, Sox2, and Nanog, which are frequently expressed in poorly differentiated tumors and are recognized as markers of tumor-initiating cells ${ }^{[13,14]}$. qRT-PCR assays revealed that after SP600125 treatment, Oct4, Sox2, and Nanog expression was clearly suppressed in KB/VCR cells, while SP600125 had no effect on the expression of these stem markers in KB cells (Figure 1A-1C). Concomitantly, SP600125 also inhibited Oct4, Sox2, and Nanog protein expression in KB/ VCR cells as revealed by Western blot analyses (Figure 1D). Similarly, we also limited JNK1/2 expression in K562/ A02 by JNK1/2 shRNA lentiviruses to inhibit JNK activity. GFP blots were used as readouts of lentivirus infection efficiency, and p-c-Jun expression served as reduction in JNK activity. we observed that limiting JNK1/2 expression by shRNA in K562/ A02 cells reduced Oct4, Sox2, and Nanog protein expression (Figure 1E).

Inhibition of JNK activity suppresses the sphere formation ability and self-renewal capacity of acquired chemoresistant cancer cells

Sphere formation and self-renewal abilities are two critical tumor-initiating cell-like properties ${ }^{[15,16]}$. We observed that limiting JNK1/2 expression in K562/ A02 cells (Figure 1E) and $\mathrm{KB} / \mathrm{VCR}$ cells (Figure 2A) significantly inhibited sphere formation in K562/ A02 cells (Figure 2B) and KB/VCR cells (Figure 2C), as reflected by fewer cellular clusters formed compared with controls, suggesting that JNK $1 / 2$ is essential for maintaining the sphere formation ability of acquired chemoresistant cancer cells. We further dissociated the cellular clusters formed by K562/ A02 cells or KB/VCR cells into single cells for measuring self-renewal capability, an ability of cancer stem cells to refresh stem cells with identical, intact potential for proliferation, expansion, and differentiation, thus maintaining the cancer stem cell pool. We determined that knocking down JNK expression significantly reduced the numbers of cellular clusters that were formed by cells dissociated from the first generation of K562/ A02 (Figure 2D) or KB/VCR (Figure 2E) spheres, indicating an inhibition of the self-renewal ability of acquired chemoresistant cancer cells by blocking JNK activity. The impact of JNK1/2 shRNA on sphere formation and selfrenewal ability was well recapitulated by SP600125, the small molecular inhibitor of JNK, in K562/ A02 cells (Figure 2F, 2G).

Blocking JNK activity inhibited the in vitro tumor-initiating ability of KB/VCR cells

To determine whether blocking JNK activity may inhibit the tumorigenic ability of acquired chemoresistant cancer cells, we used a soft agar assay and a colony assay, which are two widely used in vitro tumorigenic assays ${ }^{[17]}$. Colony formation assays revealed that KB/VCR cells formed many more colonies than the counterpart KB cells. Treatment with SP600125 clearly reduced the number of colonies formed in KB/VCR cells, while SP600125 had little effect on the colony formation ability of KB cells (Figure 3A). We observed that JNK1/2 
A

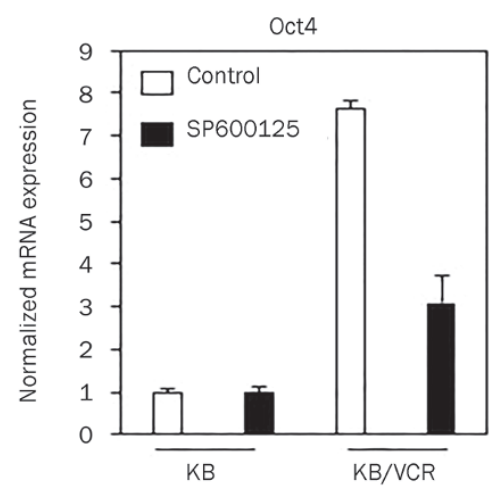

D
B

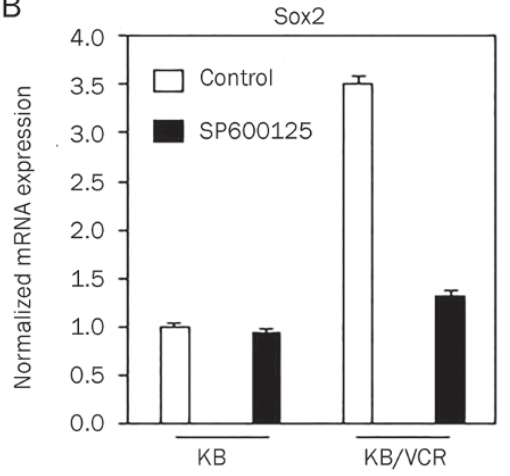

C

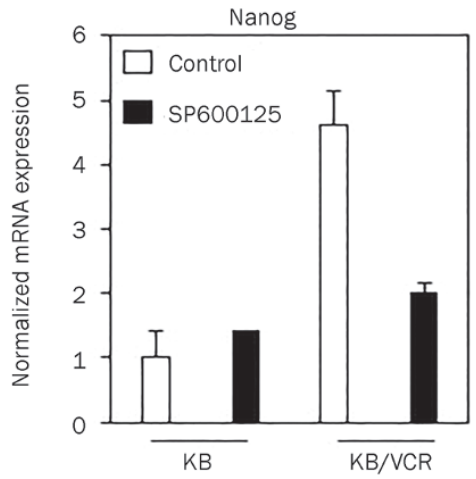

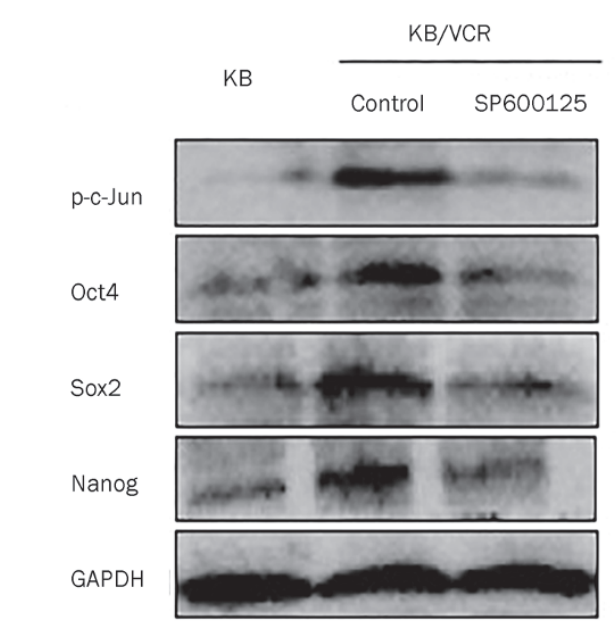

$E$

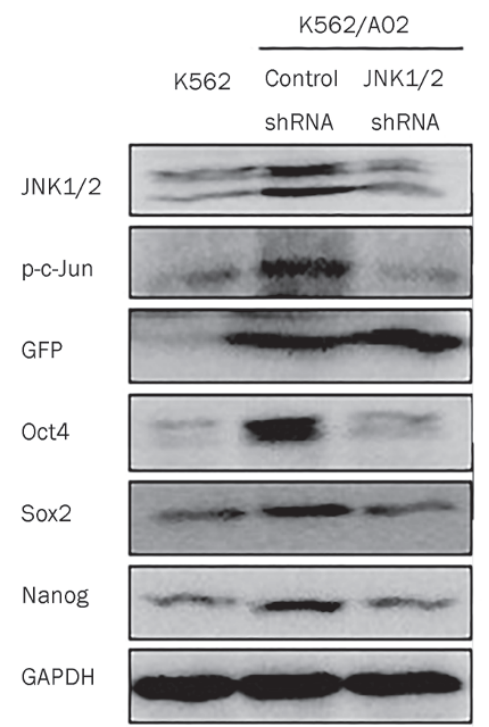

Figure 1. Inhibition of JNK activity suppresses stem cell markers of acquired chemoresistant cancer cells. (A-C) After treatment with or without SP600125 (10 $\mathrm{mmol} / \mathrm{L})$ for $24 \mathrm{~h}, \mathrm{~KB}$ and KB/VCR cells were collected for qRT-PCR and Western blot analyses. Treatment with SP600125 reduced the expression of Oct4 (A), Sox2 (B), and Nanog (C) at the mRNA level. (D) Western blot analysis of p-c-Jun, Oct4, Sox2, and Nanog expression in KB and KB/VCR cells treated with or without SP600125. (E) JNK, p-C-Jun, GFP, Oct4, Sox2, and Nanog immunoblots in K562/A02 cells that had been infected with shRNA control or JNK1/2 shRNA lentiviruses. Mean \pm SD. $n=3$.

knockdown also suppressed the colony formation ability of KB/VCR cells (Figure 3B). Furthermore, colonies formed by $\mathrm{KB} / \mathrm{VCR}$ cells in an anchorage-independent situation were also suppressed by SP600125 exposure, while SP600125 treatment exhibited no effect on KB cell colony formation in the anchorage-independent situation (Figure 3C). We further confirmed this observation by limiting JNK1/2 expression with JNK1/2 shRNA lentivirus in KB/VCR cells (Figure 3D). Hence, these observations suggest that blocking JNK activity may inhibit the in vitro tumorigenic ability of acquired chemoresistant cancer cells.

\section{Blocking JNK activity inhibits the in vivo tumor-initiating ability of $\mathrm{KB} / \mathrm{VCR}$ cells}

To test the effect of blocking JNK activity on the in vivo tumorigenic ability of acquired chemoresistant cancer cells, we infected KB/VCR cells with lentiviruses harboring JNK1(APF), a dominant negative mutant of JNK (JNK DM $)^{[18]}$. Inhibition of c-Jun phosphorylation served as inhibition efficiency of JNK DM (Figure 4A); GFP or Flag immunoblots were used as a readout of GFP or JNK DM expression (Figure 4A). We xenografted subcutaneously KB/VCR cells $\left(2 \times 10^{3}\right)$ that had been infected with lentiviruses harboring GFP or JNK DM into nude mice. The result demonstrated that KB/VCR cells infected with lentiviruses harboring GFP formed 8 tumors of 12 injections, whereas only one and two tumors were generated by KB/VCR cells that had been infected with lentiviruses harboring JNK DM and JNK1/2 shRNA, respectively (Figure $4 \mathrm{~B})$, suggesting that blocking JNK activity may inhibit the in vivo tumorigenic ability of acquired chemoresistant cancer cells.

Inhibition of JNK activity suppressed Hh activity in acquired chemoresistant cancer cells

We have previously demonstrated that the Hh signaling pathway is required for maintaining tumor-initiating cell-like 
A
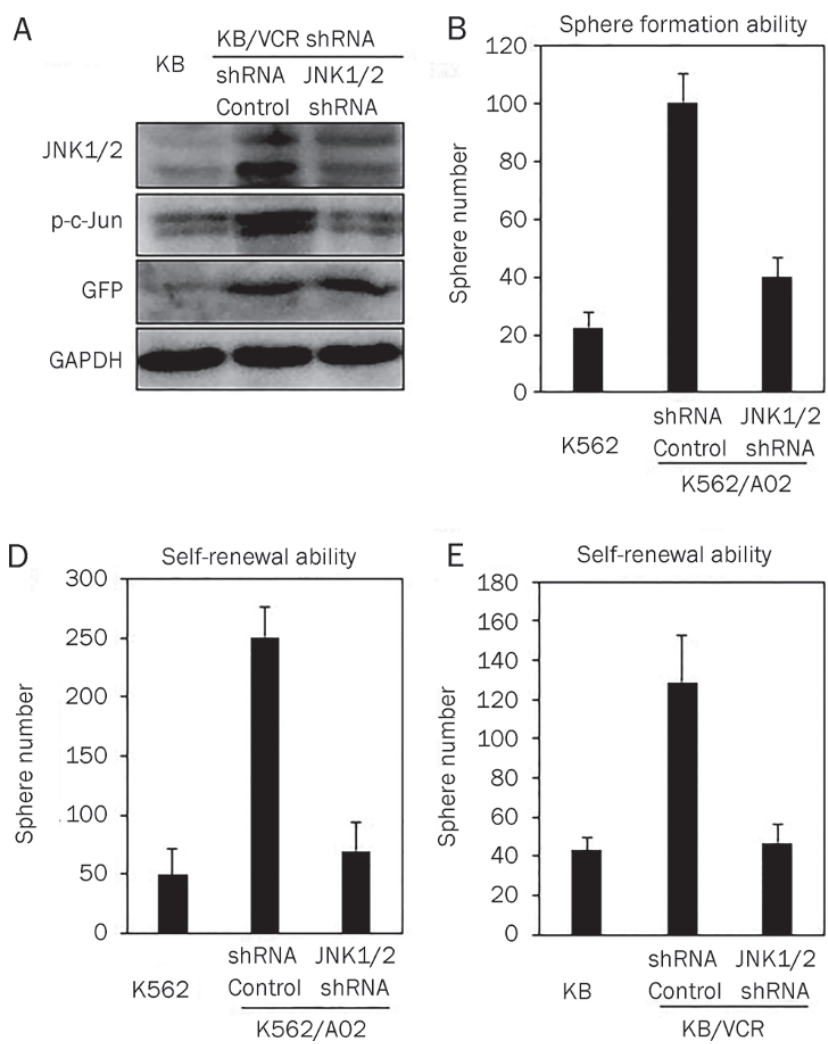
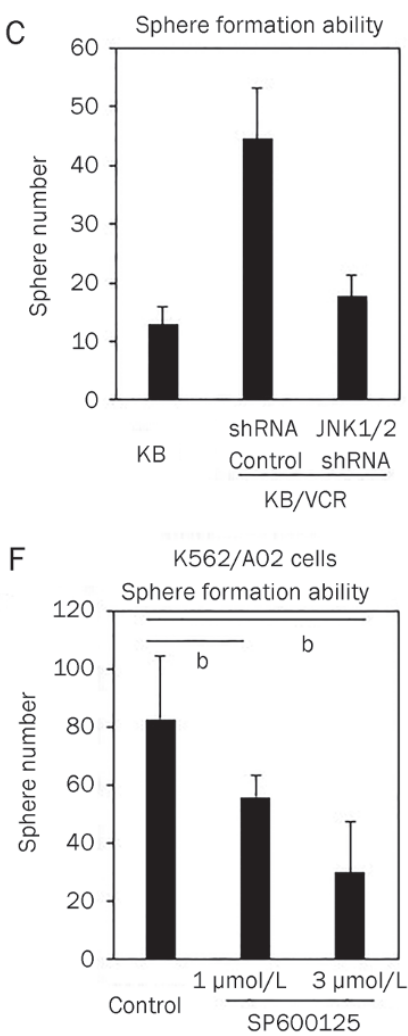

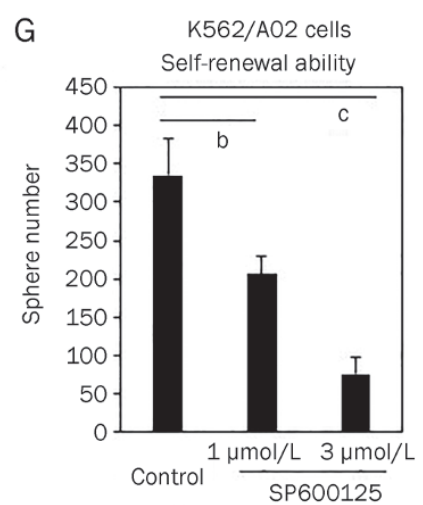

Figure 2. Inhibition of JNK activity suppressed the sphere formation ability and self-renewal capacity of acquired chemoresistant cancer cells. (A) JNK, p-C-Jun, and GFP immunoblots of KB/VCR cells that had been infected with shRNA control or JNK1/2 shRNA lentiviruses. GAPDH was used as a loading control. Sphere formation (B) and self-renewal ability (D) of K562/A02 cells that were infected with shRNA control or JNK1/2 shRNA lentiviruses. Sphere formation $(C)$ and self-renewal ability $(E)$ of KB/VCR cells that had been infected with shRNA control or JNK1/2 shRNA lentiviruses. Exposure of SP600125 inhibited sphere formation (F) and the self-renewal abilities (G) of K562/A02 cells. Mean \pm SD. $n=3 .{ }^{b} P<0.05,{ }^{C} P<0.01$.

properties of acquired chemoresistant cancer cells ${ }^{[6]}$. In this context, we asked whether JNK maintained the tumor-initiating cell-like properties of acquired chemoresistant cancer cells by activating the Hh pathway. As anticipated, we observed that suppressing JNK activity in K562/A02 cells by SP600125 clearly inhibited the mRNA expression of Gli1 (Figure 5A) and Ptch1 (Figure 5B), which are two transcriptional target genes of Hh signaling pathway transcription factors and are often used as readouts of Hh pathway activity ${ }^{[19]}$. These observations were further confirmed by limiting JNK1/2 expression with JNK1/2 shRNA lentiviruses in KB/VCR cells (Figure 5C and 5D). We further examined Hh pathway activity using dual Gli-luciferase reporter assays in acquired chemoresistant cancer cells after SP600125 treatment. As demonstrated in Figure 5E and 5F, SP600125 treatment reduced Gli-luciferase activity in K562/A02 cells (Figure 5E) and KB/VCR cells (Figure 5F). Hence, our data suggest that JNK inhibition may suppress $\mathrm{Hh}$ pathway activity, thereby disrupting the tumor-initiating celllike properties of acquired chemoresistant cancer cells.

\section{Discussion}

In this study, using two well-established acquired chemoresistant cancer cell lines K562/A02 and KB/VCR and their paren- tal counterparts, we observed that targeting JNK may disrupt the tumor-initiating cell-like properties of acquired chemoresistant cancer cells such as inhibition of pluripotent stem cell marker expression, sphere formation and self-renewal abilities, and in vitro and in vivo tumorigenic abilities. These observations suggest that JNK is required for maintaining the tumor-initiating cell-like properties of acquired chemoresistant cancer cells. We further observed that JNK activity inhibition may suppress Hh pathway activity as monitored by $\mathrm{Hh}$ pathway target genes Gli1 and ptch1 at the mRNA level as well as Gli-luciferase activity, thus indicating that JNK maintains tumor-initiating cell-like properties potentially by activating the Hh pathway. Hence, our data in the present study pave the way of abolishing tumor-initiating cell-like properties and consequently combatting acquired chemoresistance by JNK inhibition.

JNK, also termed stress-activated protein kinase (SAPK), can be activated by environmental and genotoxic stress as well as other extracellular stimuli ${ }^{[20]}$. Many studies have implicated the contribution of JNK to acquired chemoresistance. However, the role of JNK in acquired chemoresistance remains controversial, as JNK activation may promote acquired chemoresistance, or reverse acquired chemoresistance ${ }^{[9]}$. In the present 
A

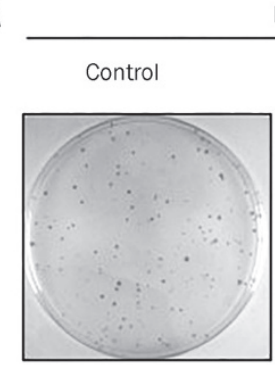

B

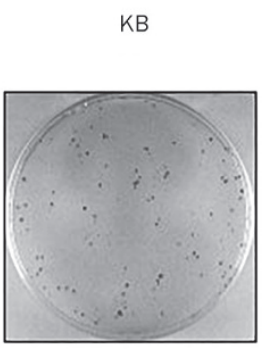

C

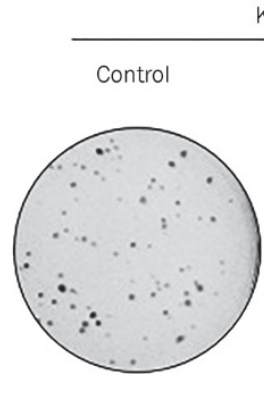

D

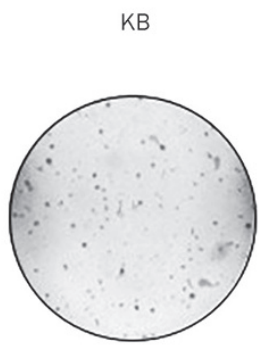

KB

SP600125

$(1 \mu \mathrm{mol} / \mathrm{L})$

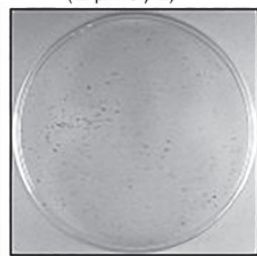

$\mathrm{KB} / \mathrm{VCR}$

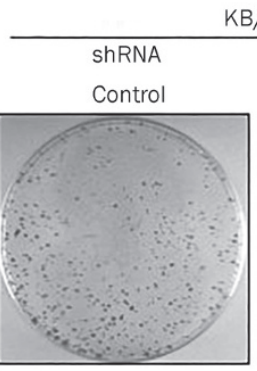

SP600125
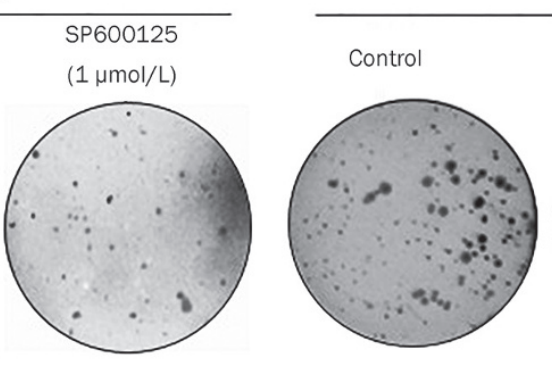

$\mathrm{KB} / \mathrm{VCR}$
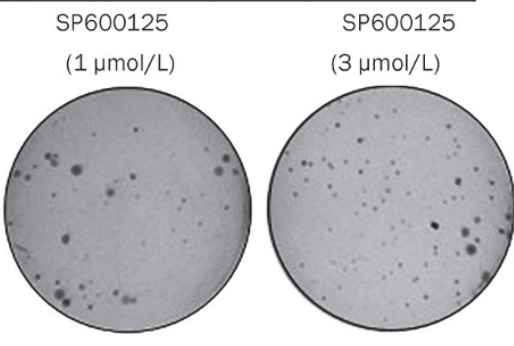

SP600125

(3 $\mu \mathrm{mol} / \mathrm{L})$

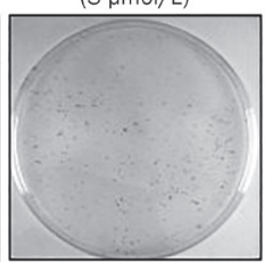

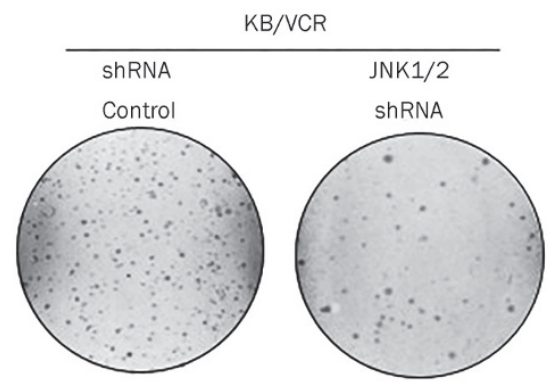

Figure 3. Blocking JNK activity inhibited the in vitro tumor-initiating ability of KB/VCR cells. (A) Colony formation of KB and KB/VCR cells that were treated with or without SP600125. (B) Colony formation of KB and KB/VCR cells that were infected with shRNA control or JNK1/2 shRNA lentiviruses. (C) Anchorage-independent colony formation in KB and KB/VCR cells after treatment with or without SP600125. (D) Anchorage-independent colony formation in KB and KB/VCR cells that were infected with shRNA control or JNK1/2 shRNA lentiviruses.

study, we determined that inhibition of JNK activity may abolish the tumor-initiating cell-like properties of acquired chemoresistant cancer cells. Thus, this finding improves the interpretation of the role of JNK in acquired chemoresistance and provides a novel mechanism underlying the contribution of JNK to acquired chemoresistance.

The Hh signaling pathway is a highly conserved pathway that ranges from insects to humans and plays a critical role for a various physiological and pathological conditions, for example embryonic patterning, maintenance of postnatal tissue homeostasis and cancer initiation and progression ${ }^{[19]}$. The
Hh signaling pathway is essential for tissue repair and cancer initiation by acting on stem cells and cancer stem cells ${ }^{[21]}$. Our prior study demonstrated that Hh signaling pathway inhibition may disrupt tumor-initiating cell-like properties and subsequently circumvent acquired chemoresistance ${ }^{[6]}$. This study suggests a critical role of the Hh signaling pathway for maintaining the tumor-initiating cell-like properties of acquired chemoresistant cancer cells. In this study, we observed that inhibition of JNK activity can suppress Hh signaling pathway activity, thus suggesting a role for JNK in activating the Hh pathway. These observations support that disrupting the 
A

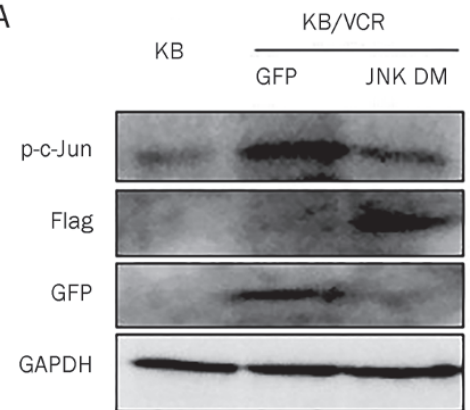

B

\begin{tabular}{ccccc}
\hline & & \multicolumn{3}{c}{ KB/VCR } \\
\cline { 3 - 5 } & KB & GFP & JNK DM & $\begin{array}{c}\text { JNK1/2 } \\
\text { ShRNA }\end{array}$ \\
\hline $\begin{array}{c}\text { Number of } \\
\text { injections }\end{array}$ & 12 & 12 & 12 & 12 \\
$\begin{array}{c}\text { Tumors } \\
\text { incidence }\end{array}$ & $1^{\text {b }}$ & 8 & $1^{\text {b }}$ & $2^{\text {b }}$ \\
\hline
\end{tabular}

Figure 4. Blocking JNK activity inhibited the in vivo tumor-initiating ability of acquired chemoresistant cancer cells. (A) Expression of phosphorylated C-Jun, Flag, and GFP in KB/VCR cells after infection with GFP or JNK DM lentiviruses. (B) Tumor formation of $K B$ or $K B / V C R$ cells after infection with lentiviruses harboring GFP, JNK DM, or JNK1/2 shRNA. Mean \pm SD. $n=3 .{ }^{\mathrm{b}} \mathrm{P}<0.05$.

tumor-initiating cell-like properties caused by JNK inhibition may be due to Hh pathway activity suppression. It warrants further investigation to elucidate how JNK promotes Hh pathway activity, which is currently being investigated in our laboratory.

\section{Acknowledgements}

This work was financially supported by National Natural Science Foundation of China (81173077).

\section{Author contribution}

Wen-fu TAN designed research, analyzed the results and wrote the manuscript; Yuan LIU, Xia ZHANG, Juan WANG, and Jun YANG performed experiments, analyzed results and contributed to writing the manuscript.

\section{References}

1 Lage $\mathrm{H}$. An overview of cancer multidrug resistance: a still unsolved problem. Cell Mol Life Sci 2008; 65: 3145-67.

2 Baguley BC. Multiple drug resistance mechanisms in cancer. Mol Biotechnol 2010; 46: 308-16.

3 Dean M, Fojo T, Bates S. Tumour stem cells and drug resistance. Nat Rev Cancer 2005; 5: 275-84.

4 Xin $\mathrm{H}$, Kong $\mathrm{Y}$, Jiang $\mathrm{X}$, Wang $\mathrm{K}$, Qin $\mathrm{X}$, Miao $\mathrm{ZH}$, et al. Multi-drugresistant cells enriched from chronic myeloid leukemia cells by Doxorubicin possess tumor-initiating-cell properties. J Pharmacol Sci 2013; 122: 299-304.

5 Calcagno AM, Salcido CD, Gillet JP, Wu CP, Fostel JM, Mumau MD, et
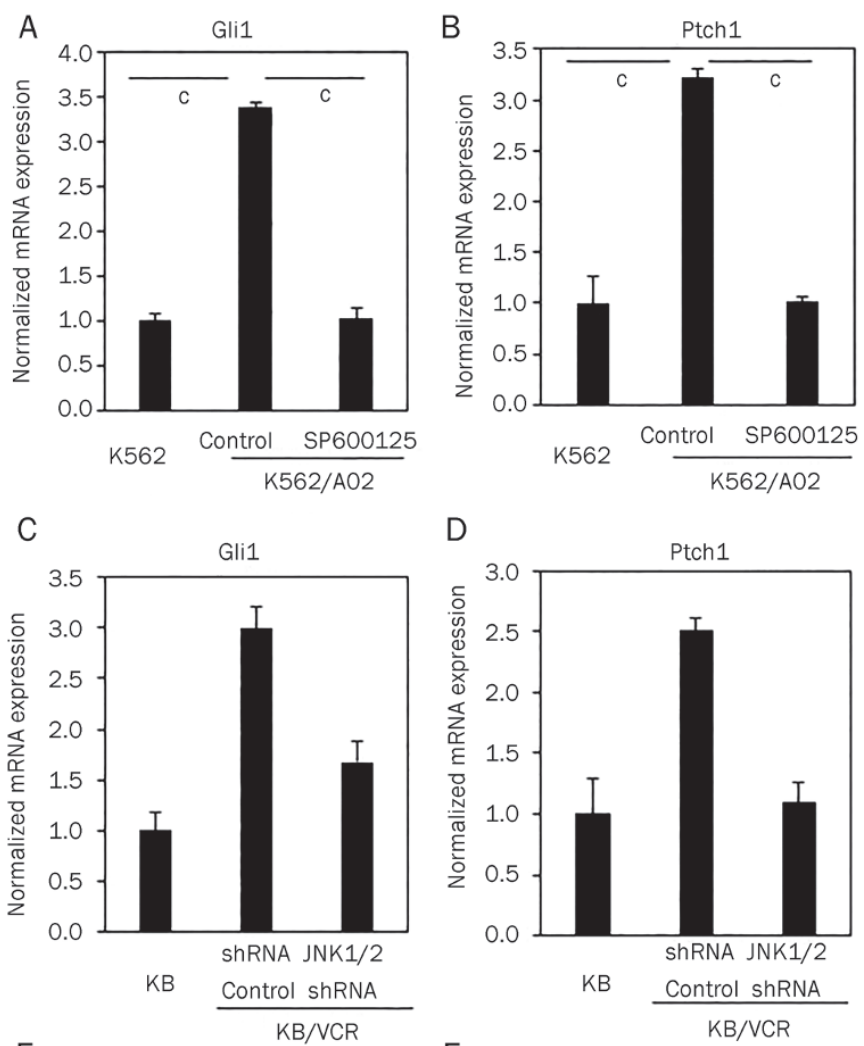

D
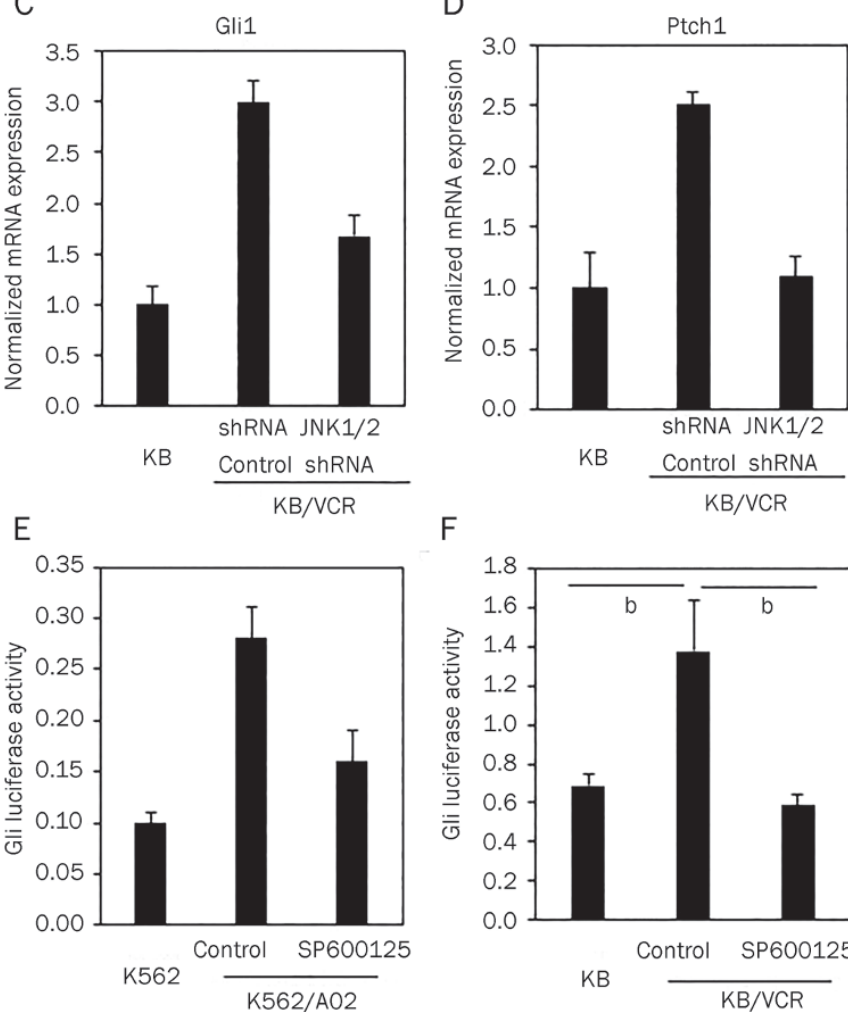

$\mathrm{F}$

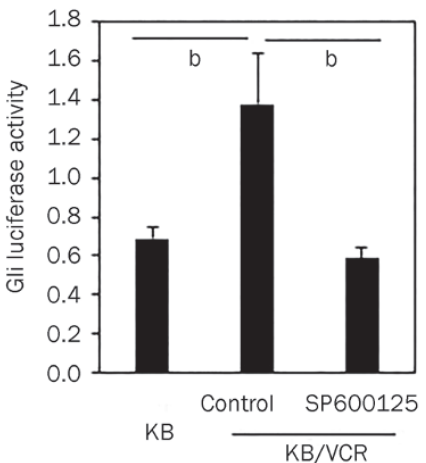

Figure 5. Inhibition of JNK activity suppressed Hh activity in acquired chemoresistant cancer cells. (A, B) After treatment with or without

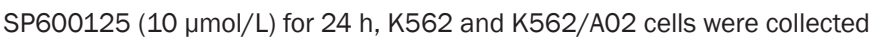
for qRT-PCR analyses. SP600125 treatment reduced Gli1 (A) and Ptch1 (B) mRNA expression. (C, D) Knockdown of JNK1/2 expression using shRNA lentiviruses suppressed Gli1 (C) and Ptch1 (D) mRNA expression in KB/VCR cells. (E, F) Inhibition of JNK activity by SP600125 reduced $\mathrm{Hh}$ pathway activity in K562/A02 cells (E) KB/VCR cells (F) as reflected by Gliluciferase reporter activity. Mean \pm SD. $n=3 .{ }^{b} P<0.05,{ }^{\mathrm{C}} P<0.01$.

al. Prolonged drug selection of breast cancer cells and enrichment of cancer stem cell characteristics. J Natl Cancer Inst 2010; 102: 1637-52.

6 Kong Y, Peng Y, Liu Y, Xin H, Zhan X, Tan W. Twist1 and Snail link Hedgehog signaling to tumor-ilnitiating cell-like properties and acquired chemoresistance independently of $A B C$ transporters. Stem Cells 2015; 33: 1063-74.

7 Davis RJ. Signal transduction by the JNK group of MAP kinases. Cell 2000; 103: 239-52. 
8 Wagner EF, Nebreda AR. Signal integration by JNK and p38 MAPK pathways in cancer development. Nat Rev Cancer 2009; 9: 537-49.

9 Vasilevskaya I, O'Dwyer PJ. Role of Jun and Jun kinase in resistance of cancer cells to therapy. Drug Resist Updat 2003; 6: 147-56.

10 Zhan X, Wang J, Liu Y, Peng Y, Tan W. GPCR-like signaling mediated by smoothened contributes to acquired chemoresistance through activating Gli. Mol Cancer 2014; 13: 4.

11 Mani SA, Guo W, Liao MJ, Eaton EN, Ayyanan A, Zhou AY, et al. The epithelial-mesenchymal transition generates cells with properties of stem cells. Cell 2008; 133: 704-15.

12 Bennett BL, Sasaki DT, Murray BW, O'Leary EC, Sakata ST, Xu W, et al. SP600125, an anthrapyrazolone inhibitor of Jun N-terminal kinase. Proc Natl Acad Sci U S A 2001; 98: 13681-6.

13 Kong D, Banerjee S, Ahmad A, Li Y, Wang Z, Sethi S, et al. Epithelial to mesenchymal transition is mechanistically linked with stem cell signatures in prostate cancer cells. PLoS One 2010; 5: e12445.

14 Ben-Porath I, Thomson MW, Carey VJ, Ge R, Bell GW, Regev A, et al. An embryonic stem cell-like gene expression signature in poorly differentiated aggressive human tumors. Nat Genet 2008; $40: 499$ 507.
15 Ponti D, Costa A, Zaffaroni N, Pratesi G, Petrangolini G, Coradini D, et al. Isolation and in vitro propagation of tumorigenic breast cancer cells with stem/progenitor cell properties. Cancer Res 2005; 65: 5506-11.

16 Dalerba P, Cho RW, Clarke MF. Cancer stem cells: models and concepts. Annu Rev Med 2007; 58: 267-84.

17 Shin SI, Freedman VH, Risser R, Pollack R. Tumorigenicity of virustransformed cells in nude mice is correlated specifically with anchorage independent growth in vitro. Proc Natl Acad Sci U S A 1975; 72 : 4435-9

18 Derijard B, Hibi M, Wu IH, Barrett T, Su B, Deng T, et al. JNK1: a protein kinase stimulated by UV light and Ha-Ras that binds and phosphorylates the c-Jun activation domain. Cell 1994; 76: 1025-37.

19 Briscoe J, Therond PP. The mechanisms of Hedgehog signaling and its roles in development and disease. Nat Rev Mol Cell Biol 2013; 14 : 416-29.

20 Huang P, Han J, Hui L. MAPK signaling in inflammation-associated cancer development. Protein Cell 2010; 1: 218-26.

21 Beachy PA, Karhadkar SS, Berman DM. Tissue repair and stem cell renewal in carcinogenesis. Nature 2004; 432: 324-31. 